\section{$\underset{\substack{\text { hommes } \\ \text { \& migrations }}}{ }$}

\section{Hommes \& migrations}

Revue française de référence sur les dynamiques

migratoires

$1285 \mid 2010$

L'appel du pied

\title{
Camel Zekri et le diwân de Biskra
}

\section{François Bensignor}

\section{(2) OpenEdition \\ Journals}

\section{Édition électronique}

URL : http://journals.openedition.org/hommesmigrations/1208

DOI : 10.4000/hommesmigrations. 1208

ISSN : 2262-3353

\section{Éditeur}

Musée national de l'histoire de l'immigration

\section{Édition imprimée}

Date de publication : 1 mai 2010

Pagination : 188-194

ISSN : 1142-852X

\section{Référence électronique}

François Bensignor, "Camel Zekri et le diwân de Biskra », Hommes \& migrations [En ligne], 1285 | 2010, mis en ligne le 29 mai 2013, consulté le 22 septembre 2020. URL : http://journals.openedition.org/ hommesmigrations/1208; DOI : https://doi.org/10.4000/hommesmigrations.1208

Ce document a été généré automatiquement le 22 septembre 2020.

Tous droits réservés 


\title{
Camel Zekri et le diwân de Biskra
}

\author{
François Bensignor
}

1 Titulaire d'un premier prix de guitare classique et d'un diplôme de musicologie, Camel Zekri a grandi en France, où s'est forgée sa réputation dans le domaine des musiques improvisées et des rencontres expérimentales. Mais sa curiosité et sa culture musicales l'ont empêché de s'enfermer dans un seul domaine d'expression. Il figure aujourd'hui parmi les rares passeurs éclairés qui savent faire le lien entre la profondeur de musiques de traditions orales portant le témoignage de grandes civilisations passées et l'expression globalisée des musiques actuelles. Cette personnalité hors du commun, il a su l'affirmer dans son travail de direction artistique auprès de musiciens africains pétris de leurs cultures traditionnelles, comme les chanteuses Hasna El Becharia d'Algérie et Malouma de Mauritanie, ou les groupes Mamar Kassey du Niger et Oudaden du Maroc.

2 Cette aptitude particulière pour appréhender le champ des traditions musicales orales, Camel Zekri la doit probablement à son grand-père, disparu au milieu des années quatre-vingt-dix, le raïs Hamma Moussa, maître de cérémonie du diwân de Biskra. Patriarche de la famille Temtaouï, il était le dernier à en maîtriser tous les éléments, dont le hejmi, langue ancienne utilisée dans les parties chantées du rituel. Ayant servi autrefois à commercer entre le nord et le sud du Sahara, le hejmi est fait de mots tirés des différentes langues des peuples habitant ces régions: Bambara, Zerma, Haoussa, Peul, etc. Le répertoire de Biskra est très riche en expressions et mots issus de cette langue, mais on en a perdu le sens et l'on ne les chante plus qu'en onomatopées. Alors que son grand-père s'apprêtait à suspendre définitivement son activité à la tête du diwân de Biskra, Camel Zekri décidait d'enregistrer dans leur intégralité les deux cérémonies qui le composent, l'une diurne, l'autre nocturne. À partir de ces enregistrements, il allait porter cette forme algérienne du rituel popularisé par les Gnaoua du Maroc à la connaissance des amateurs de musiques du monde. Une initiative qui a eu pour effet de perpétuer en la dynamisant la pratique ancestrale du diwân à Biskra, laquelle aurait tout aussi bien pu disparaître.

3 Avec l'installation récente d'un Centre dédié aux praticiens des grandes traditions musicales qui se sont maintenues jusqu'à nos jours à Biskra, grande cité oasis aux 
portes du désert, une nouvelle étape se dessine dans la démarche patrimoniale et artistique de Camel Zekri.

\section{Le diwân}

4 À l'origine de notre "divan" - sur lequel le patient du psychanalyste est en quelque sorte convié à évoquer les conflits entre les esprits qui l'habitent - le terme arabe "diwân" se réfère à la poésie écrite rassemblée en recueils, aux lieux où elle est dite, à la pièce où l'on se réunit pour débattre d'idées. À Biskra, et plus généralement en Algérie, le "diwân" est aussi le nom donné à l'équivalent des cérémonies menées par les Gnaoua au Maroc et par les Stâmbali en Tunisie. On retrouve les mêmes structures de musiques et de danses dans les pratiques cérémonielles de ces confréries familiales. Celles-ci perpétuent l'héritage de rites animistes propres aux populations subsahariennes razziées par les Berbères et les Arabes afin de servir leurs propriétaires dans une Afrique du Nord islamisée. Les relations étroites que ces groupes à peau sombre entretiennent avec le monde des génies se manifestent à l'occasion des rituels de guérison, au cours desquels les patients, entraînés par la musique et les chants, font l'expérience de la transe, guidés par des femmes initiées.

\section{Particularités et instruments}

5 Tout comme la langue, la musique, les chants et les danses accompagnant les cérémonies ont évolué de manières différentes dans chacun des pays du Maghreb. Au Maroc, on peut constater une certaine unité des pratiques chez les Gnaoua. Un phénomène que Camel Zekri attribue à la structuration politique du pays en royaume. " Cette unité n'est pas la règle en Algérie, où chaque diwân s'est perpétué dans une certaine autarcie au sein de chacune des différentes régions, développant ses propres particularités, explique-t-il. À l'Ouest, les pratiques sont très proches de celles des Gnaoua du Maroc. À l'Est, où se trouve Biskra, elles ressemblent beaucoup plus au stâmbali des Tunisiens, avec lesquels nous partageons aussi la langue. Les instruments des deux traditions se ressemblent : le guembri, luth à trois cordes emblématique joué par le maître de cérémonie, le maâlem, est rond, alors qu'il a une forme allongée et rectangulaire à l'Ouest et au Maroc, où on le nomme aussi "hajouj". À Biskra, cet instrument a également la particularité de n'apparaître que dans le diwân nocturne, la cérémonie diurne étant accompagnée des seules percussions."

"Le diwân de Biskra utilise un instrument qui lui est exclusif, le qouretou, petite percussion ressemblant à un pot de terre recouvert d'une peau de chèvre et que l'on joue avec de fines baguettes en olivier, poursuit Camel Zekri. L'architecture des percussions pour le répertoire de jour est constituée d'un qouretou, qui produit des fréquences très aiguës, et de trois autres tambours, alors qu'ailleurs, notamment au Maroc, il n'y en a souvent que deux." Ce sont des tambours circulaires tendus de peaux sur les deux faces et que l'on joue avec des bâtons recourbés. Le petit, appelé "kolo", joue dans l'aigu, le moyen, "sereh", dans le medium et le grand, "benga" dans les graves. Le mot "benga" est également utilisé pour désigner le rituel. À ces quatre percussions s'ajoutent les fameux crotales métalliques qarqabou (pluriel de qraqeb), dont s'accompagnent les chanteurs et qui jouent un rôle central dans les rituels des trois pays. 


\section{L'importance des femmes}

7 La famille qui a la maîtrise du diwân ne l'organise que si on l'a sollicité. "Il peut s'agir d'apporter une sorte de bénédiction chez soi, ou bien d'avoir recours à la thérapeutique liée au diwân, explique Camel Zekri. Dans ce cas-là, la personne va rencontrer une femme du diwân, qui lui concocte des herbes susceptibles d'apporter la guérison. Et si la guérison ne vient pas, alors on appelle les hommes afin d'organiser la cérémonie. Le diwân touche les personnes qui l'ont demandé ainsi que leurs proches, qui à Biskra constituent un public essentiellement féminin. Dans le diwân, les hommes jouent des instruments et chantent. Tout le reste, c'est-àdire une partie importante du dispositif, est de la responsabilité des femmes : l'organisation de la cérémonie comprenant le thé et les dattes, la danse, le lien avec la personne qui vient danser et qui est possédée."

8 La 'arifa (c'est-à-dire "celle qui sait") est chargée d'établir et de gérer la relation entre les femmes de l'assistance et le rituel qui se déroule aux sons des musiques et des chants. Durant tout le temps de la cérémonie, elle veille à ce que brûle l'encens, qu'elle fait circuler, afin de purifier le lieu et les personnes de l'assemblée. Le plus souvent, il s'agit d'une pièce carrée, sans meubles. Les musiciens s'installent sur un côté, l'assistance se répartissant le long des trois autres murs, afin de laisser l'espace central dégagé pour la danse. Pendant que le kanoun, d'où s'élève la fumée de l'encens, circule dans l'assistance, des femmes s'avancent pour danser. La 'arifa leur recouvre la tête d'un voile et les guide. Elle fera arrêter la musique lorsqu'une femme possédée s'écroulera au sol. Elle l'aidera à se relever, puis à retrouver ses esprits, pendant que la cérémonie reprendra son cours. "Le rôle de la 'arifa est aussi important, voire plus important que celui du maâlem, le maitre de cérémonie", affirme Camel Zekri.

\section{Le renouveau du diwân}

9 Au début des années quatre-vingt-dix, l'Algérie s'enflamme. Dans ce contexte malsain de guerre civile, le raïs Hamma Moussa décide d'arrêter le diwân. Camel Zekri en est profondément choqué. “À l'époque, j'étais en faculté d'ethnomusicologie et je voulais étudier le diwân. Mais, bizarrement, mes professeurs ne voulaient pas me laisser travailler sur mon sujet. Alors, plutôt que d'aller étudier les Indiens d'Amérique du Sud, j'ai abandonné mes études pour aller m'occuper du diwân... Depuis des années, je prenais des notes auprès de mon grandpère. Je le faisais pour mon plaisir, sans penser à la musicologie. Cela faisait partie de mon patrimoine familial. Je ne pouvais pas rater un diwân: j'adorais ça. Mais je n'avais jamais imaginé qu'un jour je m'en occuperais. J'avais mon activité de musicien en France, que je ne connectais pas avec l'Algérie. C'est à la suite de la décision de mon grand-père que je lui ai demandé si l'on pouvait enregistrer. Il ne m'a dit ni oui, ni non. Je suis reparti en France et suis revenu avec un ingénieur du son. Nous avons rassemblé mon grand-père, mes oncles et mes cousins pour enregistrer le répertoire complet de jour et de nuit. Alors tout le monde est venu, les femmes comme les hommes. J'avais demandé de le faire pour l'enregistrement, mais c'est devenu un vrai diwân, avec les danses et tout le rituel. J'ai même failli tomber en transe: c'était très fort!"

10 De cet enregistrement réalisé en 1993 sera extraite la matière d'un album, publié sur le label Ocora/Radio France en 1996. Reconnaissance de cette musique algérienne et consécration du travail de Camel Zekri, la même année le diwân de Biskra est invité à se produire à Paris sur la scène de l'Institut du monde arabe. "Entre-temps, mon grand-père 
est décédé, se souvient-il. Mes grands-mères et tous mes oncles sont venus: c'était très émouvant. Sur la scène, ils faisaient un vrai diwân. Les gens venaient danser et les organisateurs me demandaient de les faire arrêter de jouer..."

11 L'expérience de 1996 dessine un nouveau projet. Camel Zekri réunit toutes les branches de sa famille. Dans chacune d'elles, au moins un homme est choisi afin de constituer un groupe capable de présenter en concert la musique du diwân de Biskra sur les scènes internationales. "Nous avons travaillé sur le plan musical en enregistrant ce que nous faisions, afin que chacun puisse se rendre compte du son produit par l'ensemble: une sorte d'éducation par l'écoute", dit Camel Zekri.

\section{Expériences au Sud et au Nord}

Afin de compléter la culture musicale et d'élargir l'horizon artistique des membres de ce nouvel ensemble, Camel Zekri conduit ses cousins aux sources de leur musique, à la rencontre des musiciens des pays du Sud du Sahara. "Au Mali, nous avons rencontré le guitariste Djelimady Tounkara. Au Niger, nous avons retrouvé mes amis du groupe Mamar Kassey. Nous sommes allés en Mauritanie, où nous avons rencontré la chanteuse Malouma. Nous sommes aussi allés dans l'Ouest algérien voir la joueuse de guembri et chanteuse Hasna El Becharia, héritière du diwân de Bechar. Et au Maroc, j'ai invité plusieurs fois à jouer avec nous Majid Bekkas, célèbre dans le monde entier pour sa version intelligemment modernisée de la musique des Gnaoua... Ce tour nous a permis de nous interroger sur la question de la racine africaine du diwân et a considérablement enrichi et renforcé notre approche de cette tradition."

Le groupe s'est également confronté aux musiques contemporaines d'Europe, en travaillant notamment avec l'Ensemble Ars Nova, ainsi qu'aux musiques improvisées à travers des expériences de rencontres avec des musiciens de jazz. Depuis sa fondation en 1996, le groupe n'a jamais cessé de jouer et d'expérimenter de nouvelles formes de rencontres. Chaque année, le diwân de Biskra se produit en Europe. Et s'il n'a pas publié d'album depuis l'enregistrement qui a présidé à sa constitution, il n'est pourtant pas en reste en termes d'expérience de studio, comme l'explique Camel Zekri : "Parfois, plutôt que de recevoir le peu d'argent qu'une ville peut offrir en échange d'ateliers pour le jeune public, je préfêre demander du temps de studio dans un équipement géré par la ville. J'ai ainsi pu accumuler beaucoup d'heures d'enregistrements, qui nous ont permis de construire une technique musicale très performante." Certains d'entre eux vont constituer la matière d'un tout prochain disque.

\section{Les effets induits}

La reconnaissance et l'activité pérenne du diwân de Biskra dans les circuits internationaux de musique ont eu des effets très positifs. "Nul n'est prophète en son pays" dit le proverbe, ainsi ses succès à l'étranger ont considérablement renforcé la notoriété du diwân de Biskra dans sa ville d'origine. La demande a tellement augmenté qu'il existe aujourd'hui deux diwân à Biskra. Le rapport à la transmission s'est également transformé dans les familles. Lors des tournées en France, les musiciens algériens ont été amenés à communiquer leur savoir dans des ateliers de formation. De retour en Algérie, ils ont commencé à former des jeunes, pratique qui n'existait pas dans la société traditionnelle, où le musicien doit révéler ses dons par lui-même. Par ailleurs, précise Camel Zekri : "les concerts à l'étranger ont permis à mes cousins de faire 
construire leurs maisons. En une tournée, ils parviennent à gagner l'équivalent d'un an de salaire. Chacun garde son activité professionnelle en Algérie. Je leur ai fait comprendre qu'il est bien plus avantageux pour eux de rester vivre à Biskra."

\section{Un Centre musical à Biskra}

En 2010, Camel Zekri franchit un nouveau palier dans son investissement personnel en faveur des musiques patrimoniales, avec la réalisation de son projet de Centre musical dans la ville de Biskra. Il est situé dans une palmeraie au milieu de laquelle est dressée une très belle khaïma, tente berbère pouvant accueillir jusqu'à deux cents personnes.

“Pour moi, Biskra est située au centre de l'Algérie, dit-il. En une journée, on peut aller visiter les sites romains des Aurès, admirer des sites troglodytes magnifiques ou encore s'avancer dans le désert jusqu'à El-Oued. Biskra se trouve à $2 \mathrm{~h}$ de Paris, comme de Niamey. C'est une situation assez privilégiée: les gens du Sud peuvent y venir sans trop de problème et ceux du Nord y accéder facilement. Se trouvant en Afrique, Biskra bénéficie d'une temporalité différente. Obtenir un visa pour l'Algérie pose moins de problèmes aux artistes africains avec lesquels je souhaite travailler. Les coûts de production y sont moins élevés qu'en Europe. Et les diffuseurs européens seront très heureux de venir à Biskra écouter et voir si un projet peut les intéresser."

Le Centre s'inscrit aussi dans le prolongement de sa démarche familiale. "Après avoir monté le groupe, avoir permis aux musiciens de gagner leur vie grâce aux tournées, il fallait ancrer cette activité dans le territoire de Biskra, affirme Camel Zekri. C'est le rôle du Centre, autour duquel pourront se développer des activités associées, comme la facture instrumentale, la cuisine, la couture, etc. Mais aussi tout ce qui sert à créer et alimenter les relations avec l'international: Internet, un équipement informatique permettant de communiquer avec les réseaux intéressés." Pas seulement réservé au diwân, le Centre s'ouvre aux autres familles de traditions musicales anciennes de Biskra: les sonneurs de cornemuses, les joueurs de flûtes berbères et les ensembles de chants religieux soufis. Ces quatre familles sont conviées aux Nuits musicales organisées dans le Centre.

Camel Zekri précise que cette initiative strictement privée fonctionne de manière autonome. "Je ne m'inscris ni dans un cadre commercial, ni dans une forme de politique culturelle. Dans cette aventure, je reste à l'échelle humaine. Les musiciens que j'invite vivent à Biskra. Mon grand-père les a tous connus de son vivant et chacun d'entre eux pratique la musique dans le cadre de cérémonies. Jusqu'à présent je n'ai organisé que des Nuits musicales, mais l'objectif est d'ouvrir le lieu à ces familles qui, comme la nôtre auparavant, vont chez les gens faire des cérémonies, mais n'ont pas un lieu où pratiquer. Dans un premier temps, j'ai donc l'intention d'ouvrir le Centre aux cérémonies traditionnelles qui se font à Biskra. Les Nuits servent à faire connaître l'endroit, à donner l'habitude aux musiciens appartenant à ces familles de se côtoyer et de jouer ensemble. Quant au public, il est le même que celui qui traditionnellement sollicite les cérémonies: ce sont les femmes." "J'organise les Nuits comme je le ferais pour un mariage. Je rémunère les musiciens, mais je ne fais pas payer de droit d'entrée. J'offre aussi le repas. Ma vie professionnelle se déroulant ailleurs, je n'ai pas d'enjeu économique particulier lié à ce Centre. Mon objectif est essentiellement de créer de l'activité dans ce lieu, en espérant qu'à partir des habitudes d'échanges qui vont se prendre puissent naître d'autres choses. Le Centre vient d'ouvrir avec les premières Nuits musicales. De petites choses s'installent, la dynamique est lancée." 


\section{BIBLIOGRAPHIE}

\section{Discographie}

Le Diwân de Biskra (Ocora/Radio France)

Camel Zekri, producteur et coordinateur d'enregistrements du patrimoine musical du Niger :

Épopées Zarma et Soghay par Jibo Baje (Ocora/Radio France)

Musique Dendi par Harouna Goge (Ocora/Radio France)

Chasseurs du Dallol Mawri (Ocora/Radio France)

Camel Zekri, producteur artistique des albums :

Denké-denké de Mamar Kassey (Daqui)

Djazair Johara de Hasna El Becharia(Indigo / Label Bleu)

Dunya de Malouma (Marabi)

\section{RÉSUMÉS}

Alors que, parmi les signataires de la charte de l'Unesco sur la sauvegarde du patrimoine culturel immatériel, de nombreux pays tardent à mettre leurs politiques culturelles en conformité avec les textes signés, certaines initiatives privées d'artistes ouvrent la voie. La démarche entreprise depuis près de vingt ans par le musicien Camel Zekri pour permettre la survie harmonieuse, dans un contexte moderne, de la pratique traditionnelle du diwân à Biskra, est un exemple riche d'enseignement pour ceux qui souhaitent préserver une pratique patrimoniale sans la couper de son assise populaire. Retour sur une aventure à échelle humaine. 